\title{
La phýsis como problema en el fragmento 9 de Sobre la filosofía
}

\author{
CLAUDIA MARISA SEGGIARO \\ Universidad de Buenos Aires \\ Consejo Nacional de Investigaciones Científicas y Técnicas
}

Resumen: En el presente trabajo, nos centraremos en el análisis de las críticas a Parménides y Meliso realizadas en el fragmento 9 de Sobre la filosofía. Creemos que este fragmento es un testimonio relativamente sólido de que en esta obra Aristóteles habría intentado indagar en torno a uno de los principios de lo real: la phýsis, presentando las problemáticas que su negación presupone. Para realizar nuestro análisis, dividiremos el trabajo en dos partes. En la primera, examinaremos el contexto en el cual aparece citado el fragmento 9 de Sobre la filosofía y la fuente por la cual nos ha llegado: Sexto Empírico. En la segunda parte, nos centraremos en las estrategias argumentativas que Aristóteles habría implementado para desarrollar las tesis presentadas en el fragmento en cuestión.

Palabras clave: procedimiento dialéctico, principios, inmovilistas, antifísicos. 


\title{
The Phýsis as a Problem in Fragment 9 of On Philosophy
}

\begin{abstract}
In the present work, we will focus on the analysis of Parmenides' and Melissus' criticisms made in fragment 9 of On Philosophy. We believe that this fragment is a relatively solid testimony that in this work Aristotle would have tried to inquire around one of the principles of the real: the physis, presenting the problems that its denial presupposes. To perform our analysis, we will divide the work into two parts. In the first, we will examine the context in which the fragment 9 of On philosophy and the source from which it has reached us is mentioned: Sextus Empiricus. In the second part, we will focus on the argumentative strategies that Aristotle would have implemented to develop the thesis presented in this fragment.
\end{abstract} physics.

Key-words: dialectical procedure, principles, immobilists, anti-

n un trabajo anterior (2015: 1-24), hemos analizado el uso de las
opiniones en Sobre la filosofía. Allí, habíamos establecido que el uso de las opiniones de los predecesores en esta obra no cumple un rol doxográfico, sino que obedece al procedimiento dialéctico implementado para discurrir en torno a los primeros principios. ${ }^{1} \mathrm{El}$ análisis de los diferentes fragmentos conservados nos permitió establecer que hay dos tipos de opiniones que Aristóteles retoma de sus antecesores: 1) aquellas que son conflictivas, pero útiles para presentar una aporía y explicitar un problema (fragmentos 9 y 11) y 2) aquellas que tienen alguna especie de sustento observacional y que podrían calificar como éndoxa relevantes (fragmentos 12-13).

En el presente trabajo, nos centraremos en el análisis de las críticas a Parménides y Meliso realizadas en el fragmento 9 de Sobre la filosofía. Creemos que este fragmento es un testimonio relativamente sólido de que en esta obra Aristóteles habría intentado discurrir en torno a uno de los principios de lo real: la phýsis, presentando las problemáticas que su negación presupone. Para ello, Aristóteles habría tomado como punto de partida las creencias de aquellos que, al negar el movimiento, se ven forzados a rechazar la existencia de la phýsis: Parménides y Meliso. Este abordaje es un indicio relativamente claro del procedimiento dialéctico implementado por Aristó-

\footnotetext{
${ }^{1}$ Tal como veremos más adelante, esto permite trazar una continuidad entre esta obra y los dos primeros libros de la Física. Esta continuidad es subrayada por Aristóteles en Física 194a26-36.
} 
teles para argumentar en torno a una noción que encarna el principio de la generación y destrucción de las entidades sensibles.

Para realizar nuestro análisis, dividiremos el trabajo en dos partes. En la primera, examinaremos el contexto en el cual aparece citado el fragmento 9 de Sobre la filosofía y la fuente por la cual nos ha llegado: Sexto Empírico. En la segunda parte, nos centraremos en las estrategias argumentativas que Aristóteles habría implementado para desarrollar las tesis presentadas en el fragmento en cuestión. Creemos que ambas cuestiones están relacionadas, pues, al citar el extracto que habría pertenecido a Sobre la filosofía, Sexto, en cierto sentido, también trae a colación el modus operandi implementado por el Estagirita, siendo el pasaje de su obra un testimonio no solo de las tesis defendidas por Aristóteles, sino también de su procedimiento argumentativo.

\section{La trasmisión del fragmento 9 de Sobre la filosofía}

To que nos ha llegado como fragmento 9 de Sobre la filosofía es Lun pasaje de Contra los profesores (10,45-46) de Sexto Empírico. Por cuestiones exegéticas transcribiremos el párrafo en el cual se trasmite el fragmento 9, en lugar de citar solo el extracto en cuestión:

Pero, antes de pasar a las aporías, debemos tener presente que han sido tres, a grandes rasgos, las posturas acerca del movimiento. Unos, efectivamente, afirman que existe movimiento, otros que no existe, y otros, en fin, que lo mismo existe que no existe. Afirman que existe tanto la gente corriente, que se fía de las apariencias, como la mayoría de los filósofos fisicos, por ejemplo, los seguidores de Pitágoras, Empédocles y Anaxágoras, así como los de Demócrito y Epicuro, y también suscribieron esta opinión los peripatéticos e igualmente los estoicos y muchísimos otros. Que no existe, en cambio, lo sostienen los seguidores de Parménides y Meliso, a quienes Aristóteles ha llamado "inmovilistas de la naturaleza" y "antifísicos": "inmovilistas" por la inmovilidad que sostienen, y "antifísicos" porque la naturaleza es principio de movimiento, y ellos la suprimieron al afirmar que nada se mueve; en efecto, lo que se mueve debe recorrer una cierta distancia, pero toda distancia, por el hecho de admitir división hasta el infinito, es interminable, de manera

\footnotetext{
${ }^{2}$ El uso de la negrita es nuestro. Con ello, pretendemos destacar lo que suele citarse como el
} fragmento de Sobre la filosofía del resto del discurso de Sexto. 
que no existirá nada que se mueva. Finalmente, quienes sostuvieron que el movimiento lo mismo existe que no existe fueron los escépticos, porque, en cuanto respecta a las apariencias, existe un cierto movimiento, pero, en cuanto respecta al discurso filosófico, no existe (trad. Mortos Mantiel).

El contexto en el cual está inserto el fragmento de Sobre la filosofía es la discusión acerca de la existencia del movimiento. El punto de partida de la indagación de Sexto Empírico es la clasificación aristotélica de los diferentes tipos de movimiento, así como las reclasificaciones posteriores (Contra los profesores 10, 37-38). Su estrategia es partir de la postura de quienes aceptan la existencia del movimiento para luego refutarla. No obstante, antes de hacer esto, manifiesta que en relación con esta cuestión existen, al menos, tres concepciones: las de quienes admiten la existencia del movimiento, la de aquellos que lo niegan y la de los que no se pronuncian al respecto. Al introducir la tesis de quienes niegan el movimiento, cita a Aristóteles. Esto es un indicio de que la formulación de la contraposición entre movilistas e inmovilistas, que se plantea en el fragmento 9 de Sobre la filosofía, no necesariamente formaba parte del argumento de Aristóteles, sino que podría haber sido solamente un primer momento de la discusión de Sexto en torno al movimiento. Ahora bien, ¿debemos interpretar que Sexto cita a Aristóteles solo a los fines de introducir la tesis de Parménides y de Meliso?

Pese a que Sexto es una de las fuentes para la reconstrucción del pensamiento de Parménides, ${ }^{3}$ algunos autores, como por ejemplo Coxon ([1986] 2009: 5), han problematizado en torno a qué tipo de acceso tuvo a la obra de este pensador. Lo relevante es que, pese a que Coxon considera que Sexto no contaba con un manuscrito del poema de Parménides, en ningún momento evalúa la posibilidad de que se haya servido de la obra de Aristóteles como fuente. Según este autor, para la reconstrucción de las tesis del eléata, Sexto se habría valido de un tratado helenístico que contenía citas de él. Desde su perspectiva, la prueba de esto es el carácter estoico de su exégesis. Para Coxon, dicha fuente podría haber sido Posidonio.

En cuanto a Meliso, si bien la mayoría de los fragmentos que se consideran auténticos son trasmitidos por Simplicio en su Comentario a la Física de Aristóteles, el tratamiento que hace Aristóteles de este autor en su obra hace que sea una fuente no desdeñable para la reconstrucción de su pensamiento. Sin embargo, esto no significa que debamos concluir que Sexto dependía del Estagirita para poder acceder al pensamiento de este filósofo.

\footnotetext{
${ }^{3}$ Algunos de los fragmentos adjudicados a Parménides, por ejemplo, DK B1, solo nos han llegado a través de la obra de Sexto Empírico.
} 
Por otra parte, es evidente que Sexto tenía conocimiento de las tesis defendidas por Aristóteles. ${ }^{4}$ En este sentido, es relevante notar que en el pasaje citado de Contra los profesores Sexto no retoma del texto del Estagirita solo la tesis de Parménides y de Meliso, sino también el argumento por el cual esta tesis resulta inadmisible para Aristóteles. Esto nos lleva a pensar que Sexto no solo cita a Aristóteles para reponer la tesis inmovilista, sino también para apropiarse de la interpretación aristotélica que conduce a su refutación. ${ }^{5}$ Para ello, se vale de una estrategia argumentativa en cierto sentido similar a la implementada por el Estagirita para abordar esas mismas tesis. ${ }^{6}$ En este sentido, creemos que, al traer a colación la tesis inmovilista de Parménides y de Meliso a partir de la crítica de Aristóteles, Sexto no solo pone en evidencia su propio proceder metodológico, sino que también nos da algunas pistas del aristotélico.

En cuanto al modus operandi de Sexto Empírico, ${ }^{7}$ Hankinson (2015: 218) sostiene que la "estructura arquitectónica" de su argumento se expresa claramente en Contra los profesores 10, 69:

Y muchísimas otras cosas de esta índole suelen decir los de la parte contraria a favor de la existencia del movimiento. Por nuestra parte, una vez que con esto nos baste como consenso suficiente para establecer este punto de vista, vamos a ocuparnos del punto de vista contrario. Porque si se muestra que la inexistencia del movimiento es, en cuanto a credibilidad y no credibilidad, igual que su existencia, sin duda se seguirá el rechazo a apoyar cualquiera de las dos alternativas y la suspensión del juicio respecto a ambas (trad. Martos Montiel).

Si bien está analizando otra sección del primer libro de Contra los profesores $(9,195,330)$, dado que la estrategia argumentativa implementada por Sexto parece ser la misma, podemos aplicar aquí el análisis de White (2015:

\footnotetext{
${ }^{4}$ Según Annas 1992: 203, los textos de Sexto no evidencian un estudio profundo de los escritos de Aristóteles. Annas 1992: 205 argumenta que en algunos casos Sexto se basa en doxografías helenísticas, que modificaban las ideas de Aristóteles para que se ajusten a una agenda filosófica helenística. Dichas modificaciones a menudo tenían una impronta estoica, incluso después del resurgimiento del estudio textual aristotélico iniciado por Andrónico.

${ }^{5}$ Véase Física $185 \mathrm{a} 1-187 \mathrm{a} 10$ y Metafísica $986 \mathrm{~b} 10-31$.

${ }^{6}$ Un ejemplo de esto es el primer libro de la Física.

${ }^{7}$ Dado los límites del presente trabajo no podremos hacer un análisis del método de Sexto. Nuestro objetivo es más limitado: nos interesa establecer si el pasaje de Contra los profesores, fragmento 9 de Sobre la filosofía, puede leerse como un testimonio de las estrategias argumentativas implementadas por el Estagirita en su obra perdida. Para un análisis de la dialéctica en Sexto, véase Algra e Ierodiakonou 2015.
} 
74-105). Según este autor, el argumento empleado por Sexto en Contra los profesores es dialéctico en el sentido de que asume un oponente que niega la existencia de la noción a analizar, para luego refutarlo a través de una reducción al absurdo. Si tomamos como ejemplo la noción de movimiento analizada en Contra los profesores 10, podemos aplicar la interpretación de White del siguiente modo: Sexto parte de la posición sostenida por algunos pensadores, entre ellos Aristóteles, los estoicos y epicúreos, según la cual el movimiento existe. Estos serían los contrincantes que, al presentar una postura dogmática, hay que refutar. Para ello, toma como punto de partida la definición de movimiento $(10,50-61)$ y los argumentos esgrimidos por algunos pensadores que sostienen la tesis opuesta, como, por ejemplo, Diodoro de Cronos $(10,85-97 ; 101-120)^{8}$ y Zenón de Elea. ${ }^{9}$ El objetivo de esto no es adoptar esta última tesis, sino también negarla $(10,168)$, mostrando las consecuencias indeseables que su aceptación implica.

Según Algra e Ierodiakonou (2015: 25), el motivo por el cual Sexto procede de este modo es que desactivar dialécticamente las posiciones dogmáticas o una subclase bien elegida de ellas es un procedimiento menos rígido, que suele ser suficiente para inducir la suspensión del juicio. Para estos autores, esto se debe a que la suspensión del juicio, a la que nos quiere conducir Sexto, no es producto de conclusiones lógicas, sino que es una condición 362 I superviniente a lo largo de la investigación emprendida, la cual implica procedimientos psicológicos o incluso retóricos, cuyo fin es la persuasión.

Algunos intérpretes, entre ellos White (2015: 77-78) y Hankinson (2015: 245, n. 65 y 66), sostienen que los argumentos esgrimidos por Sexto tienen el "estilo dialéctico" de algunos megáricos, como Estilpón o Diodoro. ${ }^{10}$ White encuentra esta impronta megárica al analizar las objeciones

\footnotetext{
${ }^{8}$ En este caso, Sexto simultáneamente refuta los argumentos de Diodoro, sosteniendo que estos son sofismas. Véase Contra los profesores 10, 99; 102; 118.

${ }^{9} \mathrm{Si}$ bien no lo nombra expresamente, la lectura de Contra los profesores 10, 87-88 tiene algunas reminiscencias del fragmento DK 29 B 4 de Zenón de Elea. Contra los profesores 10, 142 ss. parece suponer las tesis atribuidas a este pensador en DK 29A 25. Véase también Simplicio, Comentario de la física de Aristóteles, 1013, 4-16 y Filópono Comentario de la física de Aristóteles, 81, 7.

${ }^{10}$ White 2015: 78 aclara que, al hacer esta relación con los megáricos, solo pretende remarcar que Sexto Empírico, al igual que estos pensadores, se vale de un tipo de argumento abstracto, inteligente e impactante en cuanto a su conclusión, que, sin embargo, no resulta realmente convincente, sino que despierta la sospecha de que debe haber algo erróneo. Ahora bien, para este autor, no es obvio si este modus operandi incluye argumentos basados simplemente en desacuerdos entre dogmáticos sobre algún asunto no evidente o si implica cualquier tipo de argumento en donde parece haber alguna inconsistencia lógica / conceptual.
} 
contra los que niegan la noción de causa. ${ }^{11}$ Para White (2015: 78), este argumento (Contra los profesores 9, 205-6) se puede formular del siguiente modo: si existe alguna causa, entonces existe una causa; si no es el caso de que exista alguna causa, entonces existe una causa. La razón de esto último es que el que rechaza la existencia de causas tiene para ello alguna razón (hypó tinos aitías), motivo por el cual admite involuntariamente la existencia de la noción que dicen negar. ${ }^{12}$

Lo relevante a los fines de nuestro trabajo es que, para implementar este procedimiento, Sexto toma como punto de partida de su análisis concepciones antagónicas en torno al tema a discutir. Tras hacer eso comienza el análisis exhaustivo de cada una de esas concepciones, mostrando las aporías que estas presentan.

No sabemos cómo habría procedido Aristóteles al presentar las tesis de Parménides y de Meliso en Sobre la filosofía. Sin embargo, dado que el texto se conserva de forma completa, tenemos cierta certeza sobre el modo de proceder de Aristóteles en Física I, texto que habría sido paralelo a Sobre la filosofía, en cuanto al contenido: el tratamiento de los primeros principios. ${ }^{13}$

En relación con esto último, cabe destacar que algunos autores, por ejemplo, Untersteiner (1963: 91-98), sostienen que en Sobre la filosofía se habrían analizado muchas de las tesis desarrolladas en el primer libro de la Física, sobre todo, en los capítulos 7-9. De hecho, para este autor, sacadas algunas interconexiones que se pueden trazar con los capítulos precedentes, los capítulos 8 y 9 de Física I serían relativamente autónomos. Estos habrían sido incorporados posteriormente a los efectos de formar un todo. La obra de la cual habrían sido tomados es, según Untersteiner (1963: 96), Sobre la filosofía.

\footnotetext{
${ }^{11}$ Alejandro, Sobre las Refutaciones sofísticas, 171.17-20 (Wallies): “¿Es posible que el mismo hombre mienta y diga la verdad al mismo tiempo? No. Pero, por lo tanto, el que dice "miento", miente y dice la verdad al mismo tiempo. Entonces es falso afirmar que no es posible que el mismo hombre diga la verdad y mienta al mismo tiempo" (trad. Mársico). Al comparar la estructura argumentativa de este argumento con algunos expuestos por Sexto, podemos notar el parecido al que alude White 2015: 78, al hablar de la impronta megárica de Sexto (ver nota 10). Véase Cicerón, Académicos primeros, II.29.95-30.97. Para una interpretación de los argumentos megáricos véase Wheeler 1983: 287-295; Dorion 1995: 47-52, Müller 1985: 15-18.

${ }^{12}$ Para un análisis de este argumento véase White 2015: 78 ss..

13 "Ciertamente, sobre estos principios, dice Aristóteles, ya hemos hablado anteriormente en la Física; y luego anuncia que habla sobre ello de nuevo en el libro alfa minúscula (de la Metafísica) y que plantea los problemas en torno a ellos y los resuelve en los libros Sobre la filosofía" (Ross, T. 4, Asclepio Comentario de la Metafísica de Aristóteles 112, 16-19).
} 
Al igual que Vallejo Campos (2005: 270), tendemos a creer que esta tesis no se puede justificar. No obstante, acordamos con Untersteiner (1963: 96) que la crítica a los predecesores, pero sobre todo a los eléatas, atestiguada en ambas obras, es una prueba de que en el fragmento 9 de Sobre la filosofía se abordan temáticas que están estrechamente vinculadas con las desarrolladas en Física I.

Aceptada la vinculación entre estas dos obras aristotélicas, es relevante notar que el modus operandi para indagar sobre los principios en la Física es similar a la implementada por Sexto en Contra los profesores 10:1) presentación de las posturas existentes (Aristóteles Física I 2, Sexto Contra los profesores 10, 50-65); ${ }^{14}$ 2) formulación de las aporías que cada una de estas concepciones suponen (Física I, 2-5) ${ }^{15}$ y 3) enunciación de las conclusiones, que en el caso de Aristóteles, a diferencia de Sexto, ${ }^{16}$ es la postulación de los primeros principios que legitiman a la fisica como ciencia y permiten sostener la posibilidad del conocimiento de las entidades sensibles (Física II 7-9). ${ }^{17}$ Ahora bien, para lograr esto último, Aristóteles no solo comienza su exposición por la tesis de Parménides y Meliso, sino que dedica a la refutación de esta postura dos extensos y complejos capítulos.

Si retomamos el apartado de Contra los profesores, donde Sexto analiza la noción de movimiento $(10,45-46)$, podremos notar que, cuando examina

${ }^{14}$ Véase también Contra los profesores $9,12$.

${ }^{15}$ Al comparar el discurso de Sexto (Contra los profesores 10, 1-36) y de Aristóteles (Física IV) en relación con el tratamiento del "lugar”, Algra 2015: 200 señala que en Física 208b1-209a2 Aristóteles toma a los fenómenos como el punto de partida de una investigación dialéctica. Les sigue la formulación de varias aporías, que nos hacen dudar acerca de la cuestión a estudiar. Sin embargo, esta aporías no son definitivas. Deben resolverse en el curso de la investigación posterior. Al mismo tiempo, deben salvarse tantos de los fenómenos iniciales como sea posible (no necesariamente todos). En Sexto, "los fenómenos aristotélicos" están insertos en un contexto argumentativo bastante diferente: se oponen a los contraargumentos, y esta oposición debería conducir a la suspensión del juicio (Algra 2015: 200).

${ }^{16} \mathrm{Tal}$ como lo destaca Hankinson 2015: 264 la estructura argumentativa desarrollada por Sexto en relación con cada tesis expuesta a favor y en contra del movimiento refleja un proceso dialéctico zigzagueante. Este modo de proceder es una muestra de que, para él, todos los argumentos son provisionales y que todos ellos se formulan para ser socavados nuevamente. Para Hankinson, en nada cambia el número de niveles en los que ocurre esto, ya que, de cualquier modo, el proceso conduce a la suspensión general del juicio.

${ }^{17}$ Como lo señala Algra 2015: 191, la discusión dialéctica implementada por Aristóteles no pretende ser solo una polémica contra otras teorías, sino un intento constructivo de elaborar una teoría viable dentro de un marco aristotélico. Pero, para aquellos dispuestos a dejar atrás ese marco, sus argumentos no son convincentes. Desde la perspectiva de Algra, cuando los argumentos aristotélicos se citan en el contexto de una crítica a sistemas físicos completamente diferentes, se ven poco convincentes y concluyentes. 
las aporías de las tesis inmovilistas (Contra los profesores 10, 85 ss.), Sexto no desarrolla, tal como esperaríamos, las tesis de Parménides y Meliso. No explicita sus supuestos, ni tampoco indica por qué no resultan aceptables. En esta instancia, le dedica un extenso espacio a la tesis de Diodoro (85 ss.), ya sea para criticar a los movilistas, ya sea para demostrar que sus argumentos son erísticos. Esto nos lleva a pensar que, al citar los epítetos con los cuales Aristóteles califica a Parménides y Meliso, Sexto trae a colación la disputa que el Estagirita sostuvo con estos pensadores en la Física y en Sobre la filosofía. Dado esto, creemos que la posición de estos filósofos queda refutada al mismo tiempo que es presentada, valiéndose para ello de la polémica que Aristóteles habría mantenido con ellos. De este modo, explícitamente Sexto expone las críticas aristotélicas a estos pensadores, pero también supone la trama argumentativa por medio de la cual Aristóteles los refutó.

En lo sucesivo intentaremos establecer por qué creemos que esa trama argumentativa que empleó Aristóteles y supone Sexto es dialéctica.

\section{La phýsis como problema en el fragmento 9 de Sobre la filosofía}

n Contra los profesores 10,45-46, Sexto alude a dos tesis, atribuidas
por Aristóteles a Parménides y Meliso: la primera es la negación

del movimiento y la segunda, la negación de la naturaleza. Según Sexto, Aristóteles llama a estos pensadores "inmovilistas" y "antifísicos". Ambas características se hallan relacionadas por la noción de movimiento y son, como veremos más adelante, consecuencia una de la otra. Para entender la función que habría tenido el tratamiento de estas tesis en Sobre la filosofía es clave el siguiente pasaje de la Física 194a26-36:

También es tarea de la fisica conocer "aquello con vistas a", es decir, el fin y cuantas cosas son con vistas al fin. Pero la naturaleza es fin y causa final; porque si en las cosas cuyo movimiento es continuo hay algún fin de ese movimiento, tal fin será tanto su término extremo como aquello en vista de lo cual. Por eso también el poeta burlonamente se vio inducido a decir: "tiene el fin para el que nació". En efecto, no pretende ser fin de cualquier término sino solo el mejor. Puesto que las técnicas también producen la materia - unas absolutamente, otras la adecuan a sus propósitos- nos serviremos de todo como si todas las cosas existiesen para nosotros. En efecto, en cierto sentido, también nosotros somos un fin, pues la finalidad tiene dos significados. Esta cuestión ya la hemos tratado en Sobre la filosofía (Física 194a26-36. trad. Boeri levemente modificada). 
Este pasaje es relevante por dos motivos. El primero es la vinculación que Aristóteles realiza entre movimiento, naturaleza y fin. Según Aristóteles, la generación es un tipo de movimiento que tiene un punto de partida y una meta que se debe cumplir, si no hay nada que lo impida (Física 199a8-20). Es el proceso por el cual una forma inhiere en una materia, determinándola y transformándola en un tóde $t i{ }^{18}$ Sin embargo, Aristóteles señala que la cosa no alcanza su ser en sentido pleno simplemente por poseer la forma. Para que esto suceda, debe cumplir aquellos procesos o funciones que están determinados por esta última. ${ }^{19}$ Sin embargo, desde su perspectiva, hay una diferencia entre poseer una función y ejercerla. Poseer una función implica que potencialmente puede ser realizada. ${ }^{20}$ Ahora bien, tener esa potencialidad no equivale a que la función se llevará a cabo. El ojo tiene la potencialidad de ver, porque esa es su función, pero, en el caso de algunas personas, los ciegos, esa función nunca se actualiza y, en el caso de los videntes, no siempre se está ejerciendo.

El segundo motivo por el cual el pasaje citado de la Física (294a26-36) es relevante es que en él Aristóteles remite explícitamente a Sobre la filosofía, alegando que: "estas cuestiones ya las hemos tratado" en dicha obra. Esta remisión es un testimonio de la continuidad doctrinal entre ambos escritos y, por lo tanto, de la pertinencia de aplicar a Sobre la filosofía el análisis efectuado al pasaje de la Física.

Ahora bien, en la Física Aristóteles realiza las siguientes afirmaciones que pueden ser clave para entender el rol de la crítica a Parménides y Meliso en Sobre la filosofía:

\begin{tabular}{|l|l|}
\hline Física, 185a12-17 & Física, 193a3-6 \\
\hline Damos por supuesto que las cosas & Que la naturaleza existe, sería ri- \\
que son por naturaleza, o todas o & dículo intentar demostrarlo; pues \\
algunas, están en movimiento; esto & es claro que hay cosas que son así, \\
es claro por inducción. No estamos & y demostrar lo que es claro por lo \\
obligados a refutar toda doctrina & que es oscuro es propio de quienes \\
adversa, sino solo cuantas concluyen & son incapaces de distinguir lo que es \\
falsamente de los principios de una & cognoscible por sí mismo de lo que \\
demostración; en caso contrario, no & no lo es. (trad. Echandía). \\
(trad. Echandía). & \\
\hline
\end{tabular}

\footnotetext{
${ }^{18}$ Véase Balme 1987: 283-285.

${ }^{19}$ Física 199a8-11. Para un análisis de la concepción teleológica de la naturaleza en Aristóteles, véase Cooper 1987: 243-274; Leunissen 2010; Quarantotto 2002.

${ }^{20}$ Véase Rosen 2014: 82.

${ }^{21}$ Véase Tópicos 105a3-5.
} 
La primera afirmación (Física 185a12-17) está inserta en el contexto de la crítica a la postulación eléata de un único ser y, por lo tanto, de un único principio. Desde la perspectiva aristotélica, la aceptación de un único ser tiene dos consecuencias inadmisibles: 1) la negación de la existencia de principios y, por lo tanto, de la ciencia como el conocimiento de dichos principios y 2) el rechazo del movimiento y, por ende, de la generación. En Física I, Aristóteles solo se centra en la primera de ellas. El pasaje citado de la Física es el fundamento por el cual no cree necesario detenerse en la segunda cuestión. ${ }^{22}$ Para Aristóteles, las entidades sensibles están sujetas a cambios: nacen y se corrompen, padecen alteraciones y cambian de lugar, es decir, están atravesadas por la noción de movimiento. Esto, en cierto sentido, es una evidencia. Para Aristóteles:

pretender que todas las cosas están en reposo y apoyarse para ello en la razón rechazando el testimonio de los sentidos es debilidad intelectual, y es poner en duda todas las cosas, no solo una parte.Y no solo es oponerse a los físicos (tòn physikón), sino a todas las ciencias (tás epistémas) y las opiniones (tás dóxas) recibidas, ya que todos hacen uso del movimiento (trad. Echandía, Física $253 \mathrm{a} 32-253 \mathrm{~b} 2)$.

La segunda afirmación corresponde a Física, 193a3-6. Tras haber establecido que la phýsis es principio y causa del movimiento y reposo,Aristóteles afirma que no tiene ningún sentido cuestionarse si esta existe o no existe. Al ser un principio, la phýsis es aquello por lo cual las cosas se vuelven inteligibles, no porque sea lo más evidente para nosotros, sino porque es el fundamento ontológico y epistemológico de las cosas. Para dar cuenta de las entidades sensibles o lo que es por naturaleza debemos partir del conocimiento de ese principio. ${ }^{23}$ Proceder del modo inverso sería quebrantar el principio epistemológico defendido por Aristóteles en buena parte de sus escritos. ${ }^{24}$

Ahora bien, si tal como lo indica Aristóteles no tiene sentido discutir sobre la existencia del movimiento y de la naturaleza ¿por qué en Sobre la filosofía Aristóteles trae a colación las tesis de Parménides y Meliso que implican negar aquellas nociones que considera incuestionables?

Creemos que esto último está relacionado con el modus operandi implementado por Aristóteles en Sobre la filosofía. Para analizar este modo de proceder, transcribiremos nuevamente el fragmento 9:

\footnotetext{
${ }^{22}$ Véase Física 254a23-29.

${ }^{23}$ Véase Física 253b2-6.

${ }^{24}$ Véase Analiticos posteriores 71b9-72a14 y Física 184a10-184b14.
} 
Que no existe, en cambio, lo sostienen los seguidores de Parménides y Meliso, a quienes Aristóteles ha llamado "inmovilistas de la naturaleza" y "antifísicos": "inmovilistas" por la inmovilidad que sostienen, (stasiótas apò tês stáseos) y "antifísicos" porque la naturaleza es principio de movimiento (arkhè kinéseos), y ellos la suprimieron al afirmar que nada se mueve. (trad. Mortos Mantiel)

Al introducir el pasaje en el cual aparece citado un extracto de Sobre la filosofía, señalamos que quien estaba desarrollando la problemática del movimiento era Sexto Empírico. La lectura del fragmento 9, a la luz de los otros fragmentos conservados, no nos permite establecer si Aristóteles ha tratado esta noción en Sobre la filosofía. Sin embargo, hay ciertas cuestiones desarrolladas en esos otros fragmentos que pueden ser la clave para comprender los motivos por los cuales Aristóteles habría creído pertinente el tratamiento de la tesis antimovilista de Parménides y Meliso en esta obra. La primera de ellas está relacionada con lo que habría sido el objetivo de Sobre la filosofía. Según Asclepio (Comentario de la Metafísica de Aristóteles 112, 16-19), en esta obra, tal como lo había hecho en la Física y la Metafísica, Aristóteles había problematizado en torno a los primeros principios y causas. Algo parecido sostiene Plutarco (Obra morales y de costumbres XII, Isis y Osiris 370c, fragmento 6), al señalar que Aristóteles, en contraposición a los caldeos, habría postulado la forma y la privación ${ }^{25}$ como principios y fundamentos de la generación. Plutarco no menciona la obra a la cual se está refiriendo, pero, si, tal como lo indican los editores, se puede afirmar que está comentando una tesis de Sobre la filosofía, sus palabras serían una corroboración del testimonio de Asclepio.

La segunda cuestión importante es la definición de "phýsis" como principio de movimiento del fragmento 9 de Sobre la filosofía. Esta definición prácticamente reproduce la acepción dada en Física 192b21-22 y en Acerca del cielo 268b16. En estos dos últimos textos, la phýsis es el principio intrínseco que explica la generación de las ousíai sensibles. Ahora bien, por la Física sabemos que la naturaleza, en cierto sentido, es la materia o el sustrato que subyace en todo proceso de generación (193a10-193a30), pero, en otro sentido, es la forma o configuración conceptual de la entidad sensible (193a30-193b2). Sin su intervención no sería posible explicar cómo una entidad llega a ser lo que es. La identificación de la naturaleza con estos dos principios permite entender la función causal que estos tienen en el proceso

${ }^{25}$ Para un análisis exhaustivo de las problemáticas en torno al pasaje de Plutarco (fragmento 6) véase Untersteiner (1963: 91-98). Véase también la nota 13 del presente trabajo. 
de generación (Física 193a29-193b21). Dado esto, si tomamos como punto de partida la afirmación de Asclepio, según la cual en este último escrito Aristóteles formula los problemas en torno a los principios postulados en la Física e intenta resolverlos, es esperable que la phýsis haya sido una de las nociones trabajadas en el texto. No obstante, es evidente que, cuando Asclepio afirma esto, no puede estar queriendo decir que el Estagirita ha realizado una demostración de los principios. Esto entra claramente en contradicción con las tesis aristotélicas.

Para Aristóteles, de los principios considerados en sí mismos no podemos decir nada, pues "son primeros respecto de todas las cosas" (Tópicos, 101b1). Por tal motivo, si se quiere discurrir sobre ellos, es necesario hacerlo a través de éndoxa ${ }^{26}$ concernientes a cada uno de ellos. "Ahora bien, esto es propio o exclusivo de la dialéctica: en efecto, al ser adecuada para examinar (cualquier cosa), abre camino a los principios de todos los métodos" (Tópicos, 101b3-4, trad. Candel Sanmartín).

En función de esto, si en Sobre la filosofía Aristóteles se cuestionó acerca de los principios, entre ellos la phýsis, debió haberlo planteado como un problema dialéctico.

En Tópicos, 104b1-8, Aristóteles sostiene que un problema dialéctico es una cuestión que: 1) es susceptible de ser debatida significativamente (Smith 1997: 80) y 2) suscita opiniones divergentes (Tópicos, 104b14-17). Dicho tipo de problemas puede implicar una dificultad real o ser formulado con fines instrumentales a los efectos de abordar otra cuestión respecto de la cual hay un inconveniente real (Tópicos, $104 \mathrm{~b} 1$ ss.). ${ }^{27}$

En Sobre la filosofía Aristóteles habría planteado un problema de esta última índole. Para ello, se valió de una cuestión respecto de la cual algunos pensadores (Parménides y Meliso) tenían creencias opuestas a las propias. $\mathrm{Al}$ proceder de este modo, Aristóteles formula un problema que, desde su

\footnotetext{
${ }^{26}$ En sus escritos, Aristóteles distingue los éndoxa de los ádoxa. Un éndoxon se diferencia de un ádoxon en que es una opinión ampliamente aceptada. Siguiendo una línea de lectura coherentista, Berti 2004: 273-274 alega que el criterio para atribuir a los éndoxa un valor de verdad y otorgarle a la dialéctica un valor epistemológico no es el grado de "endoxabilidad" de sus premisas, sino su consistencia, no solo con otros éndoxa, sino con la mayoría de ellos. Por tal motivo, "la coherencia de una proposición con la mayor parte de los éndoxa también es un signo de su verdad" (Berti 2004: 273). Los ádoxa, en cambio, son opiniones contrarias a las creencias habituales (Tópicos, 104b24-26).

${ }^{27}$ Siguiendo a Brunschwig 1967: XXVI se puede decir que un problema dialéctico se formula mediante una doble interrogación - ¿x es p o no es p?- o a través de una disyunción del tipo "es p o no es p". Debido a esta estructura, un problema dialéctico solo admite dos respuestas posibles, la afirmativa o la negativa, prefigurando la confrontación a la que se dirige.
} 
perspectiva, no es real -la existencia del movimiento y de la naturaleza- para dar cuenta de otra cuestión, que, de otro modo, no podría ser demostrada: la naturaleza de los primeros principios. ${ }^{28}$

En Tópicos Aristóteles sostiene que, al plantear un problema dialéctico:

1. debe haber alguien que pregunte y otro que responda. ${ }^{29} \mathrm{Si}$ esto no es posible, el mismo individuo puede adoptar los dos roles de forma alternada. ${ }^{30}$ Sin embargo, tal como lo resalta Gourinat (2002: 47), la dialéctica "no se distingue de las otras formas de argumentación por el hecho de que haya dos interlocutores que mantengan una conversación mediante preguntas y respuestas", pues este es el rasgo distintivo del diálogo. La especificidad de la dialéctica es la de ser un proceso dialógico a partir de éndoxa.

2. Si el objeto de discusión son los principios o aquellas nociones cercanas a ellos, debido a la naturaleza y dificultad de la tarea propuesta, se podrá partir de tesis menos convincentes (Tópicos, 159a3-15). Como indica Brunschwig (1967: 115), una vez insertas en el cuerpo de un razonamiento sofisticado, que conduce a una conclusión inaceptable, estas tesis revelan su carácter vicioso. Su inserción en un razonamiento de este tipo pone de manifiesto el carácter erístico que el uso de estas tesis suele tener. Desde la perspectiva de Brunschwig (1967: 115), esto último no excluye la necesidad de una cierta perspicacia para percibir la naturaleza de este vicio (Tópicos, 101a1).

3. Si bien Aristóteles explicita que, cuando se implementa la dialéctica con el fin de realizar una indagación científica, no hay reglas establecidas acerca de cómo se debe responder, establece que el interrogado debe partir de un ádoxon, de un éndoxon o de una tesis que no es ni lo uno ni lo otro (Tópicos, 158b37-39). En el primer caso, la conclusión deberá ser un éndoxon; en el segundo, un ádoxon; en el tercero, ni lo uno ni lo otro (Tópicos, 159a4 ss.). Al sostener esto, Aristóteles supone la dinámica interrogador/interrogado: si el interrogado parte de una opinión que cumple con cualquiera de estas características, ser un éndoxon o un ádoxon, el interrogador lo conducirá a concluir lo opuesto.

\footnotetext{
${ }^{28}$ En los fragmentos $8 \mathrm{~b}$ y $8 \mathrm{c}$ se apela a la metáfora para caracterizar a los primeros principios y dar cuenta de cómo estos son conocidos por nosotros. El uso de este recurso tampoco pretende ser una demostración, sino una manera de hacer comprensible aquello que de otro modo sería mucho más difícil de entender.

${ }^{29}$ Véase Gourinat 2002. Para este autor, en Refutaciones sofisticas 2, texto en el cual se dan las reglas de la dialéctica, Aristóteles emplea dialégesthai en el sentido más restringido de "dialogar mediante preguntas y respuestas" (2002: 468).

${ }^{30}$ Para un análisis de las reglas para la formulación de las preguntas y respuestas dialécticas y de los roles de los interlocutores véase Refutaciones Sofisticas 2 y Tópicos VIII 5.
} 
En este punto, cabe mencionar la distinción trazada por Brunschwig. Citando a Smith, Brunschwig (2007: 280) señala que en Tópicos, 159a18-20 la tarea del interrogador es conducir la discusión de modo tal que el interrogado se vea obligado a establecer conclusiones que resulten lo más opuestas posibles a las ideas aceptadas. En Tópicos, 158b38ss, en cambio, el fin del interrogador es construir, con las premisas que el entrevistado le otorga, un razonamiento deductivo, cuya conclusión contradecirá la tesis de este último. Si bien la primera tarea puede describirse como una refutación por el absurdo de la tesis del interrogado, la segunda puede describirse como una refutación directa de esta misma tesis. ${ }^{31}$

En lo sucesivo, intentaremos establecer si podemos rastrear estos tres requisitos básicos en el argumento del fragmento 9 de Sobre la filosofía.

En cuanto al primer ítem: ser un proceso dialógico basado en las opiniones de los interlocutores, debemos tener presente que hay una creencia relativamente unánime de que Sobre la filosofía habría sido una obra escrita en forma de diálogo, ${ }^{32}$ en la cual se presume que uno de los protagonistas habría sido Aristóteles. Si tomamos como punto de partida la estructura dialógica de la obra, se podría pensar que cada uno de sus interlocutores presentaba su tesis, y al menos algunos de los otros personajes formulaba las objeciones. Esto se desprende del hecho de que algunos de los fragmentos conservados, el 9 entre ellos, presenta las tesis de sus antecesores y sus consecuencias. En la serie de fragmentos 13 y 14, Aristóteles habría dado cuenta de cómo habrían postulado la necesidad de una divinidad quienes pensaron sobre el tema antes que él. El contenido de estos fragmentos nos hace pensar que el interlocutor del Estagirita en esta instancia es Platón, más específicamente Timeo, el personaje creado por este pensador en su obra homónima. En la serie de fragmentos 11 y 12, en cambio, solo aparecen las objeciones a algunas de estas teorías, principalmente a la teoría de las Ideas. En la serie de fragmentos

\footnotetext{
${ }^{31}$ Véase Refutaciones sofisticas, 165a2-3. Para Brunschwig 2007: 280, la diferencia entre ambas descripciones es que, en la primera, el interrogador utiliza las tesis del entrevistado como premisas, a partir de las cuales busca deducir consecuencias paradójicas, sin pretender establecer una consecuencia completamente determinada; en la segunda descripción, el interrogador debe fijar una conclusión definitiva en su razonamiento, pero no puede usar las tesis del entrevistado como premisas.

${ }^{32}$ Prisciano Lidio, Soluciones a Cosroes, 1 6-42, 3 (Rose 1; Ross, T .2) "(Las doctrinas desarrolladas) han sido extraídas y compuestas a partir del Timeo de Platón ...y de los tratados de Aristóteles, como la Física, Acerca del cielo, Acerca de la generación y la corrupción, y los Meteorológicos; y también igualmente a partir de Acerca del sueño y Acerca de los ensueños y de las obras que están escritas en forma de diálogos, como Sobre la filosofía y Acerca de los mundos" (trad. Vallejo Campos). Véase también Cicerón, Cartas a los familiares 1, 9, 23.
} 
8 , y en los fragmentos 16 en adelante, aparecen esbozadas concepciones de corte aristotélico, las cuales podrían ser interpretadas como las respuestas a los diferentes problemas abiertos en la obra. ${ }^{33}$

Teniendo esto como telón de fondo, se puede ensayar la idea de que, emulando los diálogos de corte platónico y recuperando la tradición socrático-megárica, ${ }^{34}$ en este diálogo Aristóteles habría procedido dialécticamente. ${ }^{35} \mathrm{Si}$ bien no tenemos elementos para sostener que en Sobre la filosofía haya usado la dinámica de preguntas y respuestas, los fragmentos conservados nos permiten aseverar que en esta obra Aristóteles ha presentado algunas teorías, las problemáticas planteadas a esas teorías, y sus posibles respuestas, emulando el modus operandi de la dialéctica descripto en Tópicos.

En el segundo ítem, establecimos que un problema dialéctico puede comenzar con un ádoxon, cuando lo que se pretende discutir son primeros principios. Tal como lo vimos más arriba, la contrapartida de un ádoxon es un éndoxon. La característica fundamental de un éndoxon es el consenso del cual dispone. Para Aristóteles, un éndoxon ${ }^{36}$ es una opinión aceptada por la mayoría o por los sabios (Tópicos, 100b22-23). ${ }^{37}$ En contraposición, un ádoxon es una creencia paradojal. ${ }^{38}$ Una opinión de este tipo es una tesis sostenida solo por un individuo, que, por lo general, no solo se contrapone a lo que dice la mayoría, sean estos sabios o no, sino que además contradice los fenómenos. Como ejemplos de este tipo de opiniones (Tópicos, 104b1928), Aristóteles menciona la tesis de Antístenes que niega el principio de

${ }^{33}$ En el fragmento $8 \mathrm{~b}$ trasmitido por Filópono se sostiene que la naturaleza es artífice o demiurgo de las cosas. Esta concepción de la naturaleza como principio de la generación claramente se contrapone a la postura antifísica de Parménides y Meliso, pudiendo haber sido la respuesta de Aristóteles a estos pensadores.

${ }^{34}$ Para la relación entre la dialéctica socrática y aristotélica véase Bolton 1993: 121-152 y Dorion 2011: 563-582. Ambos autores presentan tesis antitéticas.

${ }^{35} \mathrm{Al}$ igual que en el caso de Sexto Empírico, creemos que la relación entre la dialéctica aristotélica y la megárica se limita a ciertas coincidencias en el procedimiento argumentativo sobre la base del cual presenta la tesis de sus interlocutores. En este sentido, aplicamos a Aristóteles la interpretación que White 2015: 78 realiza de la dialéctica en Sexto. Véase nota 10.

${ }^{36}$ Según Brunschwig 1967: 114, una opinión no es un éndoxon por derecho propio o en virtud de su contenido intrínseco. La "endoxabilidad" es una propiedad que le pertenece de hecho. Por tal motivo, no es licito traducir éndoxon por "probable", "plausible" y otros adjetivos con un sufijo similar.

${ }^{37}$ Para esta cuestión véase Chichi 1997; Mié 2013; Owen 1961; Vega Reñón 1998.

${ }^{38}$ Brunschwig 1967: 114 señala que un enunciado paradójico no es una proposición intrínsicamente improbable o inverosímil, sino una creencia que, de hecho, ofende las opiniones prevalecientes. 
no contradicción y la opinión de Heráclito según la cual todo deviene y nada permanece. Si bien pueden ser el origen de un problema dialéctico (Tópicos, 104b1ss.), apoyar este tipo de opiniones es una simpleza (Tópicos, 104b22-24).

Las opiniones de Parménides y Meliso mencionadas en el fragmento 9 de Sobre la filosofía son las consecuencias inmediatas de aceptar un único ser y de negar el no ser en sentido absoluto (Física, 184b26 ss.). Es decir, se desprende de una de las tesis que en Tópicos, 104b19-28 Aristóteles califica de ádoxon. Si bien en el fragmento 9 la negación del movimiento y de la naturaleza no es calificada abiertamente como ádoxon, en Física, 185a5-13 Aristóteles caracteriza a quienes sostienen esta tesis como erísticos, puesto que, partiendo de premisas falsas, llegan a conclusiones igualmente falsas. Aplicando la definición de Tópicos I 1, podemos decir que la razón por la cual los argumentos de Parménides y Meliso son erísticos es que parten de premisas que parecen éndoxa, sin serlo realmente (Tópicos, 100b23-25). ${ }^{39} \mathrm{Tal}$ como lo indicamos más arriba, para Aristóteles, discutir estas creencias es un absurdo (Física, 185a12-25; 193a1-6). Pese a esto, considera que puede ser el comienzo de un problema dialéctico y el punto de partida de una indagación filosóficamente relevante (Física, 185a12-20).Al ser sostenida por filósofos que han tenido gran reputación, queda compensado su bajo grado de credibilidad (Brunschwig 1967: XXVIII). ${ }^{40}$

Esto nos introduce al tercer ítem. Tal como lo indicamos más arriba, al formular un problema dialéctico con fines epistémicos, se debe partir de un ádoxon, un éndoxon o una proposición que no sea ni lo uno ni lo otro. Si se parte de un ádoxon, se arribará a un éndoxon; si la indagación comienza por un éndoxon, se concluirá un ádoxon; si el punto de partida no es ni lo uno ni lo otro, lo mismo pasará con su conclusión.

Pese a que Sexto no nos ha trasmitido el análisis que Aristóteles habría hecho de las tesis de Parménides y Meliso en Sobre la filosofía, intentaremos reconstruir los posibles argumentos esgrimidos contra estas, de modo tal de establecer qué rol tenían en el texto.

${ }^{39}$ Véase Berti 2004: 271-273.

${ }^{40}$ Desde la perspectiva de Aristóteles, la concepción del ser de Parménides ha tenido un fuerte impacto en los filósofos posteriores, algunos de los cuales han elaborado sus teorías a partir de sus supuestos. Tal es el caso, por ejemplo, de Anaxágoras (Física, 187a26 ss). Como lo indica Curd [1998] 2004: 6, Leucipo y Demócrito también siguen el modelo parmenídeo al intentar proporcionar una explicación racional de cómo son las cosas. Afirman la unidad monogénica de cada átomo, cumpliendo los criterios parmenídeos de lo que es, y luego apelan a la mezcla y separación de átomos para explicar fenómenos sensibles. 
En el fragmento 9, Aristóteles expone dos tesis que, tal como lo indicamos, son ádoxa:

1. el movimiento no existe;

2. la naturaleza no existe.

Ambas tesis se hallan relacionadas por la noción de movimiento y son consecuencia una de la otra. Puesto que todo principio debe ser principio de algo, si se niega su efecto, se niega también su causa. Debido a esto, creemos que la primera tesis -el movimiento no existe- es el ádoxon que constituye el punto de partida del interrogatorio, mientras que la segunda tesis -la naturaleza no existe- es la conclusión a la que deben arribar los interlocutores de Aristóteles (Parménides o Meliso). El razonamiento es el siguiente: si admitimos que el movimiento no existe y que la naturaleza es principio del movimiento, estamos forzados a admitir que la naturaleza tampoco existe. ${ }^{41}$

Ahora bien, al insertar las tesis de Parménides y Meliso en una estructura argumentativa más sofisticada, el razonamiento arriba formulado pierde, desde la perspectiva aristotélica, su aparente consistencia. Ambas tesis entran en contradicción con un conjunto de creencias ampliamente aceptadas y establecidas, que, según Aristóteles, intentan explicar los fenómenos observados y hacen posible la física como ciencia (Física, 253a33-253b2). En este sentido, un modo de mostrar la inviabilidad de las tesis de Parménides y de Meliso es mostrar con cuáles creencias sus tesis son incompatibles y por qué se produce esa incompatibilidad.

Desde la perspectiva aristotélica, ${ }^{42}$ la negación del movimiento por parte de Parménides tiene su justificación en el rechazo del no ser. Si lo único que puede existir es el ser y bajo ningún punto de vista el no ser (Metafísica, 986b27-30), entonces solo puede haber un único ser, ${ }^{43}$ que

\footnotetext{
${ }^{41}$ Tal como indica Naddaf 2005: 12, la raíz de la forma nominal de la noción de phýsis es bhū, cuyo significado primario es crecimiento, producción y desarrollo. Entre los presocráticos, el término phýsis tenía cuatro significados básicos. Designaba: (1) la materia primordial; (2) un proceso; (3) la materia primordial y un proceso y (4) el origen, el proceso, y el resultado de ese proceso. Naddaf 2005: 3 destaca que, en un sentido amplio, el término permitía referir al origen y crecimiento del universo de principio a fin. La idea de movimiento era, en este sentido, inherente a la noción de phýsis, de ahí que negar la primera implicará rechazar, de alguna manera, la segunda.

${ }^{42}$ Es importante recalcar que nuestro análisis se focaliza en la lectura aristotélica de las tesis de Parménides y Meliso. Pese a esto, nuestro objetivo no es establecer si la interpretación de Aristóteles se ajusta o no a las creencias de estos pensadores, sino indagar por qué considera pertinentes traerlas a colación.

${ }^{43} \mathrm{Al}$ analizar el Poema de Parménides, Curd [1998] 2004: 4ss. sostiene que la necesidad de concluir un único ser, entendiendo por esto la posibilidad de que solo haya un ser, es la
} 
debe ser eterno, perfecto y un todo completamente homogéneo (DK B8, $22-25 ; 42-49)$. Este no puede estar sujeto a ningún tipo de cambio, sea este locativo, cualitativo o cuantitativo; pues, si así sucediese, se tendría que admitir el no ser. Claramente esto implica negar el movimiento y con ello la generación.

Al analizar esta concepción, en Acerca del cielo 298b15-20, Aristóteles sostiene que, aunque estos pensadores en relación con algunas cuestiones hablan correctamente (légousi kalôs), no hay que creer que lo están haciendo "desde un punto de vista fisico (physikôs); en efecto, que algunas de las cosas que existen sean inengendradas y totalmente inmóviles es más propio de una investigación distinta y anterior a una especulación natural" (trad. Candel Sanmartín, levemente modificada). Esta desvinculación de lo sensible es la causa por la cual en Metafísica, 986b18-20, Aristóteles señala que el discurso de estos pensadores es katà tòn lógon. ${ }^{44}$

No obstante, en Metafísica, 986b27-34, revindicando la figura de Parménides, Aristóteles recalca que este, obligado por los fenómenos (phainómena) y de acuerdo a la sensación (katà tén aísthesin), admite la existencia de la pluralidad y de dos principios materiales: lo caliente, el fuego, y lo frío, la tierra (Metafísica, 984b1-4 y Acerca de la generación y corrupción, 318b6-7, $330 b 13-15) .{ }^{45}$ En la misma línea argumentativa, en Física, 188a19-22 añade que todos sus predecesores, incluso los que sostienen que el todo es uno e inmóvil, aceptan que los principios de la generación son los contrarios, pues, entre ellos, Parménides postula como principios lo caliente y lo frío, llamándolos fuego y tierra.

A esto se debe agregar que en Metafísica, 986b10-11, Aristóteles incluye a Parménides y Meliso entre los pensadores que "proclamaron que todo

consecuencia de realizar una lectura "existencial" del verbo "es”. Para esta autora, en el caso de Parménides esta lectura es errónea. Desde su perspectiva, en el poema de Parménides "es" tiene un sentido "predicativo", razón por la cual, al decir que el ser es uno, no quiere indicar que haya un único ser, sino que una cosa debe poseer una única naturaleza (Curd [1998] 2004: 5). Mourelatos 2008: XVII habla de monismo lógico, cuya consecuencia es la caída en una especie de misticismo.

${ }^{44}$ Se debe recalcar que en esta obra y en este pasaje en particular, Aristóteles diferencia la concepción de Parménides y de Meliso, diciendo que el primero planteó lo uno katà tòn lógon, mientras que el segundo afirma la existencia de lo uno según la materia. Para el uso de la expresión katà tòn lógon aplicada a Parménides véase Díaz 2014: 140 ss..

${ }^{45}$ Según Díaz 2014: 140, “En este texto Aristóteles está realizando una lectura del texto parmenídeo volcada a sus propios esquemas conceptuales, indicando una vía según la cual el principio es uno solo y otra que sostiene la existencia de dos principios. No se refiere a ellas, (...) vía de la verdad y de la opinión, como se esperaría a partir del vocabulario parmenídeo". 
es una única naturaleza". ${ }^{46}$ En esta misma obra, Aristóteles califica a estos pensadores como un subgrupo dentro de los llamados physiológoi. Tal como lo recalca Naddaf (2005: 139-140), pese a que Parménides esté buscando un punto de partida inexpugnable, todavía piensa dentro de la tradición peri phýseos de sus predecesores, ${ }^{47}$ "una tradición cuya política y ética se basan en modelos cosmológicos" (Naddaf 2005: 139-140). ${ }^{48}$ Desde la perspectiva de Naddaf aunque se piense que Parménides ha abandonado una investigación del tipo peri phýseos para realizar un estudio exhaustivo de lo que significa que algo sea, se debe admitir que sus reflexiones se desprenden del análisis exhaustivo de las creencias de sus antecesores milesios sobre la phýsis como principio (arkhé). Es por este motivo que, tras hacer su examen sobre el ser, Parménides expone una cosmogonía que se basa, al menos parcialmente, en su análisis previo (Naddaf 2005: 113).

La lectura de los pasajes comentados o citados de la Física, de Acerca el cielo y de la Metafísica muestra cómo Aristóteles en una misma trama argumentativa ${ }^{49}$ realiza un doble movimiento. Claramente comienza analizando las tesis de Parménides y Meliso, las cuales no pueden formar parte de un discurso físico, pues niegan la generación. A pesar de esto, rescata aquellas creencias atribuidas a uno de estos pensadores, Parménides, las cuales, dada su naturaleza, pueden ser consideradas como explicaciones de este tipo. Es la relación entre estas dos clases de opiniones lo que, en parte, genera la dificultad que aparece presupuesta en Sobre la filosofía. Esta dificultad se debe a que suponer que existen dos causas del devenir (lo caliente y lo frío) implica admitir, al menos dos cosas: 1) la postulación de "entidades" que, al igual que la phýsis en el fragmento 9 de Sobre la filosofía, son principios del movimiento y 2) la existencia de la generación y, por ende, del movimiento; pues no tendría sentido postular un principio de aquello que no existe. Ahora bien, ambas cosas deben ser negadas, si se acepta como concluyente el razonamiento esbozado en el fragmento 9 de Sobre la filosofía o se admite la postura de Parménides y Meliso sintetizada en Acerca del cielo, 298b15-20

\footnotetext{
${ }^{46}$ Véase también Metafísica, 98427-984b1.

${ }^{47}$ Naddaf 2005: 135 incluso señala que la tradición antigua veía a Parménides como un physikós. Para mostrar esto, remite a Aristóteles, Física, 184b15-25; 186a11-25; y a Platón Teeteto, 152d-e; Sofista, 242c-e). Véase también Curd [1998] 2004: 6.

${ }^{48}$ En relación con esto, se debe destacar que ya desde la Antigüedad se creía que Parménides habría escrito una obra que se habría intitulado Sobre la naturaleza. Sexto Empírico, Contra los matemáticos 7.11-114, y Simplicio, Comentario a Acerca del cielo, 556, 25 (28 A 14).

${ }^{49}$ Decimos "en una misma trama argumentativa" básicamente porque en una misma indagación, el análisis de las causas en Metafísica I y el examen de los principios en Física I, Aristóteles trae a colación estas tesis contrapuestas que, en rigor, solo le atribuye a Parménides.
} 
o en Metafísica, 986b10-20. Con esto, Aristóteles estaría dando cuenta de una inconsistencia no solo en relación con los éndoxa establecidos, sino en relación con sus propias concepciones; pues, según Aristóteles, estos pensadores no creen que existan otras entidades además de las sensibles (aisthetôn ousían) (Acerca del cielo, 298b20-21), ${ }^{50}$ razón por la cual, al esgrimir cada una de sus tesis, se refieren a los mismos objetos. ${ }^{51}$

Esta crítica se agudiza, si tenemos en cuenta que Aristóteles califica el discurso de Parménides y Meliso como peri phýseos, pues de la lectura del fragmento 9 de Sobre la filosofía no se desprende que Parménides y Meliso hayan rechazado la noción de naturaleza, sino que se ven obligados a negarla, al sostener que no existe el movimiento. Ahora bien, al tener que asumir esta última postura, ambos pensadores entran en contradicción con los éndoxa admitidos por cierta tradición filosófica en la que, según Aristóteles, ellos mismos están inmersos. ${ }^{52}$

Al hacer esto, el objetivo de Aristóteles no es simplemente refutar a Parménides y Meliso. Su modus operandi habría sido elegir interlocutores que han sostenido tesis opuestas a las sostenidas por él y mostrar los problemas que estas presentan. De este modo, pretende poner de manifiesto las inconsistencias en las que estos pensadores incurren al negar las dos nociones elementales de la física: movimiento y naturaleza. Esto es un modo dialéctico de discurrir en torno a los principios de la generación.

\footnotetext{
${ }^{50}$ Se debe tener en cuenta que, al hacer esto, Aristóteles cruza en su análisis la primera parte del poema del Parménides con la segunda. Este modo de proceder es cuestionable, debido a que resulta problemático establecer qué status tiene el discurso de la segunda parte del poema y qué rol cumple en la economía del texto (Véase Curd [1998] 2004 y Mourelatos 2008). No obstante, se debe destacar que, al comentar las tesis de Parménides, el objetivo de Aristóteles no pretende ser doxográfico. Su intención es proponer un interlocutor que posea concepciones no solo opuestas a las suyas sino lo suficientemente conflictivas como para que puedan ser el punto de partida del procedimiento dialéctico. Para la aparente contradicción trazada por Aristóteles entre el lenguaje katà tòn lógon y el lenguaje según los fenómenos supuesta en el pasaje de la Metafísica véase Luchetta 2009: 186-197.

${ }^{51}$ Según Naddaf 2005: 135, Platón asoció la doctrina del ser y la unidad de Parménides con el universo físico, lo cual, desde su perspectiva, es corroborado por Aristóteles en Metafísica 986b24.

${ }^{52}$ La crítica que se le podría hacer a Aristóteles es que al hacer esto en definitiva tiene en cuenta acepciones de phýsis que, si bien no acuña él sino que fueron sostenidas por parte de la tradición filosófica, seguramente no serían las aceptadas por Parménides o Meliso. Esto hace que el resultado del proceso sea problemático. Véase nota 17.
} 


\section{Conclusiones}

— $\mathrm{n}$ este trabajo, hemos señalado que el fragmento 9 de Sobre la

Cilosofía es un testimonio de que Aristóteles habría abordado en esa obra la cuestión de los primeros principios dialécticamente. Para ello, dividimos el trabajo en dos partes. En la primera, nos detuvimos en la transmisión del fragmento. En esa instancia, intentamos demostrar que, al citar a Aristóteles, Sexto Empírico no solo repone la tesis de Parménides y Meliso, sino también la interpretación y los argumentos aristotélicos para rechazarlos. Al hacer esto, creemos que implícitamente Sexto pone al descubierto el modus operandi del Estagirita en su obra perdida.

En la segunda parte, intentamos reconstruir el método dialéctico implementado por Aristóteles en Sobre la filosofía. Nuestra tesis es que ese fragmento debe leerse como la formulación de un problema dialéctico, cuyo fin es dar cuenta del principio de la generación de las entidades sensibles, la naturaleza, que por definición no puede ser demostrado. Para ello nos centramos en el análisis de tres rasgos de un problema dialéctico: 1) la dinámica de preguntas y respuestas que supone dos interlocutores que presentan tesis antitéticas; 2) la posibilidad de iniciar un problema dialéctico a partir de una tesis paradójica o ádoxon, cuando el objeto de discusión son los primeros principios y 3) la necesidad de arribar a un éndoxon, si el punto de partida de la discusión es un ádoxon. En cuanto al primer punto, establecimos que la naturaleza dialógica de la obra y el contenido de los fragmentos conservados hace que sea plausible pensar que en ella Aristóteles haya propuesto algunas tesis de sus predecesores, las problemáticas en torno a ellas y sus posibles soluciones. En cuanto al segundo y tercer punto establecimos que las opiniones de Parménides y Meliso presentadas en el fragmento 9 de Sobre la filosofía son consideradas por Aristóteles como creencias paradójicas, ádoxon. Si bien pueden ser el punto de partida para iniciar una discusión, en función de que contradicen los phainómena y las opiniones fuertemente establecidas, sostenerlas es una banalidad.

Una de las nociones que estos pensadores se ven obligados a rechazar, para ser coherentes con la negación del movimiento, es la de naturaleza. Al hacer esto, dichos pensadores entran en contradicción con un éndoxon fuertemente establecido por la tradición filosófica, dentro de la cual, según el Estagirita, estarían inmersos. En el caso de Parménides, incluso, esta negación entraría en contradicción con otro discurso que este pensador se ve forzado a realizar katà tén aísthesin. De este modo, Aristóteles habría discurrido en torno a los principios de la física a partir de establecer las consecuencias que su negación presenta. Para ello, "discute" con aquellos que niegan estos principios e intenta establecer las aporías en las que incurren, al hacer eso. 


\section{BIBLIOGRAFÍA}

Algra, K. (2015), "Place: M 10.1-36 184", en K. Algra y K. Ierodiakonou (eds.), Sextus Empiricus and Ancient Physics (Cambridge: Cambridge University Press, 184-216).

Algra, K y Ierodiakonou, K. (2015), “Introduction”, en K. Algra y K. Ierodiakonou (eds.), Sextus Empiricus and Ancient Physics (Cambridge: Cambridge University Press, 1-32)

Annas, J. (1992), “Sextus Empiricus and the Peripatetics”, Elenchos, 1-2: 201-231.

Aristotele, I Dialoghi, introduzione, traduzione e commento cura di M. Zanatta (Milano: Biblioteca Universitaria Rizzoli, 2008).

Aristóteles, Física, introducción, traducción y notas de G. Echandía (Madrid: Gredos, 1995).

Aristóteles, Física, Libros I y II, traducción, introducción y comentario de M. Boeri (Buenos Aires: Biblos, 1993).

Aristóteles, Metafísica, introducción, traducción y notas de T. Calvo Martínez (Madrid: Gredos, 2007).

Aristóteles, Tratados de lógica (Órganon), tomo I introducción, traducción y notas de M. Candel Sanmartín (Madrid: Gredos, 1998).

Aristóteles, Tratados de lógica (Órganon), tomo II, introducción, traducción y notas de M. Candel Sanmartín (Madrid: Gredos, 1995).

Balme, D. M. (1987), “Teleology and Necessity”, en J. Lennox y A. Gotthelf (eds.), Philosophical Issues in Aristotle's Biology (Cambridge: Cambridge University Press, 275-285).

Berti, E. (2004), Nuovi studi aristotelici (Brescia: Morcelliana).

Bolton, R. (1993), "Aristotle's Account of the Socratic Elenchus", Oxford Studies in Ancient Philosophy, 11:121-152.

Brunschwig, J. (1967), Aristote, Topiques I-IV, texte établi et traduit (Paris: Les Belles Lettres).

Brunschwig, J. (2007), Aristote, Topiques V-VIII, texte établi et traduit (Paris: Les Belles Lettres).

Chichi, G. (1997), “Opinión mayoritaria ‘éndoxon’ y verdad en el diálogo aristotélico”, Revista de Filosofía y Teoría Política, 31-32: 64-75.

Cooper, J. (1987), "Hypothetical Necessity and Natural Teleology", en J. Lennox y A. Gotthelf (ed.), Philosophical Issues in Aristotle's Biology (Cambridge: Cambridge University Press, 243-274).

Coxon, A. H. [1986] (2009), The Fragments of Parmenides: A Critical Text with Introduction and Translation, the Ancient Testimonia and a Commentary (Las Vegas: Parmenides Publishing).

Curd, P. [1998] (2004), The Legacy of Parmenides Eleatic Monism and Later Presocratic Thought (Las Vegas: Parmenides Publishing). 
Díaz, M. E. (2014). “Alternativas para pensar la génesis: El mapa aristotélico de los que abordaron el problema de la generación”, en G. Marcos y M. E. Diaz (eds.), El filósofo y sus adversarios en los escritos de Platón y Aristóteles (Buenos Aires: Rhesis, 135-151).

Dorion, L. A. (1995), Aristote, Les réfutations sophistiques, introduction, traduction et commentaire (Paris:Vrin).

Dorion, L. A. (2011), “Aristote et l'élenkhos socratique”, Les études philosophiques, 99: 563-582.

Gourinat, J. B. (2002), "Dialogo y dialéctica en los Tópicos y las Refutaciones sofísticas de Aristoteles", Anuario Filosófico, 35: 463-495.

Hankinson, R. J. (2015), “Motion: M 10.37-168”, en K.Algra y K. Ierodiakonou (eds.), Sextus Empiricus and Ancient Physics (Cambridge: Cambridge University Press, 217-274).

Leunissen, M. (2010), Explanation and Teleology in Aristotle's Science of Nature (Cambridge: Cambridge University Press).

Luchetta, J. (2009), Metafisica 1. La sophia degli antichi. Vol. 1: Vailati traduce Aristotele (Lanciano: Carabra)

Mársico, C. (2014) (ed.), Filósofos socráticos: Testimonios y fragmentos I. megáricos y cirenaicos, introducción, traducción y notas (Buenos Aires: Losada).

Mié, F. (2013), “Fenómenos y creencias en Aristóteles: Una interpretación sobre el rol metodológico de los éndoxa en la ciencia natural”, Logos. Anales del Seminario de Metafísica, 46: 211-234.

Mourelatos, A. (2008), The Route of Parmenides (Las Vegas: Parmenides Publishing).

Müller, R. (1985), Les Mégariques: Fragments et témoignages (París:Vrin).

Naddaf, G. (2005), L'origine et l'évolution du concept grec de phýsis (New York: The Edwin Mellen Press).

Owen, G. E. L. (1961), “Tithénai tà phainómena”, en S. Mansion (ed.), Aristote et les problèmes de méthode (París: Béatrice Nauwelaerts, 83-103).

Quarantotto, D. (2002), Causa finale, sostanza, essenza in Aristotele: Saggio sulla struttura dei processi teleologici naturali e sulla funzione del telos (Napoli: Bibliopolis).

Rose, V. [1886] (1966), Aristotelis qui ferebantur Librorum Fragmenta (Stuttgart:Teubner).

Rosen, J. (2014), "Essence and End in Aristotle", Ancient Philosophy, 46: 73-107.

Ross, W. D. (1964), Aristotelis Dialogorum Fragmenta (Oxford: Clarendon Press).

Sánchez-Escariche, E. J. (2000), Aristóteles, Partes de los animales, introducción, traducción y notas (Madrid: Gredos).

Seggiaro, C. M. (2015), "El uso de las opiniones de los predecesores en Sobre la Filosofía", Revista de Filosofía y Teoría Política, 47: 1-24.

Sexto Empírico, Contra los profesores, introducción, traducción y notas de J. M. Mortos Mantiel (Madrid: Gredos, 2012).

Smith, R. (1987), Aristotle, Topics, Books I and VIII with excerpts from related texts (Oxford: Clarendon Press). 
Untersteiner, M. (1963), Aristotele, Della filosofia, introduccione, traduzione e commento esegetico (Roma: Edizioni di Storia e Letteratura).

Vallejo Campos, A. (2005), Aristóteles, Fragmentos, introducción, traducción y notas (Madrid: Gredos).

Vega Reñón, L. (1998) “Aristotle’s éndoxa and Plausible Argumentation”, Argumentation, 1: 95-113.

Wheeler, S. C. (1983), "Megarian Paradoxes as Eleatic Arguments", American Philosophical Quarterly, 20: 287-295.

White, M. J (2015), “Cause: M 9.195-330 74”, en K. Algra y K. Ierodiakonou (ed.), Sextus Empiricus and Ancient Physics (Cambridge: Cambridge University Press, 74-105).

Recibido: 13-05-2020; aceptado: 11-08-2020 